\title{
Avaliação da pressão intraocular através do ocular response analyzer antes e após cirurgia de facoemulsificação
}

\author{
Assessment of intraocular pressure through \\ the ocular response analyzer before and after \\ phacoemulsification surgery
}

Bruno de Freitas Valbon ${ }^{1}$; Renata Siqueira Silva²; Daniela Jardim³; Ana Laura Canedo3; Marcelo Palis Renato Ambrósio Junior ${ }^{5}$

\section{RESUMO}

Objetivo: Verificar a variação das medidas pressóricas através do Ocular Response Analyzer (ORA, Reichert): IOPg (gold standard, Goldmann correlated intraocular pressure) e IOPcc (corneal compensated intraocular pressure) antes e após a cirurgia de catarata por facoemulsificação. Métodos: Estudo observacional prospectivo envolvendo 47 olhos de 36 pacientes que se submeteram à cirurgia de catarata por facoemulsificação com implante de lente intraocular. A Pressão intraocular compensada da córnea (IOPcc) e correlacionada com Goldmann (IOPg), foram medidos pelo Ocular Response Analyzer (ORA); As medidas foram realizadas no pré-operatório, $1^{\circ}$ dia e $30^{\circ}$ dia após a cirurgia de facoemulsificação. O Teste de Kolmogorov-Smirnov foi utilizado para avaliar a distribuição normal. Diferenças obtidas entre as medidas obtidas através do Ocular Response Analyzer foram avaliadas pelo T-test (Student's paired t-test). Foi considerado como estatisticamente significante $p<0,05 \%$. Resultados: As médias da IOPcc no pré-operatório, no primeiro dia e no trigésimo dia após a cirurgia de facoemulsificação e implante de LIO foram de $15.6( \pm 3.06), 15.5( \pm 3.14)$, e 15.71 ( \pm 4.34$)$. A média de IOPg no pré-operatório foi de $14.7( \pm 3.0)$, no primeiro dia após cirurgia $12.9( \pm 3.1)$ e no trigésimo dia $13.4( \pm 3.7)$. Houve diferença significativa entre IOPg (PRE X D1) com p <0,01. Conclusão: Observamos uma diminuição da IOPg no pós-operatório imediato em córneas com edema após facoemulsificação. Logo, ressaltamos a importância da biomecânica na medida da Pio.

Descritores: Biomecânica; Pressão intraocular/fisiologia; Córnea/fisiologia; Facoemulsificação; Técnicas de diagnóstico oftalmológico

\footnotetext{
${ }^{1}$ Pós Graduando (Mestrado) da Universidade Federal Fluminense - UFF - Rio de Janeiro (RJ), Brasil;

${ }^{2}$ Fellowship em Glaucoma no Wills Eye Hospital - Philadelphia - EUA;

${ }^{3}$ Médica Assistente do Instituto de Olhos Renato Ambrósio - Rio de Janeiro (RJ), Brasil;

${ }^{4}$ Professor Adjunto da Faculdade de Medicina da Universidade Federal Fluminense - UFF - Rio de Janeiro (RJ), Brasil;

${ }^{5}$ Coordenador do Grupo de Estudos de Tomografia e Biomecânica de Córnea do Rio de Janeiro - Rio de Janeiro (RJ), Brasil.

Recebido para publicação em: 27/7/2010 - Aceito para publicação em 21/9/2010
} 


\section{INTRODUÇÃO}

$\Delta$ tonometria de aplanação por Goldmann (TAG), padrão-ouro para a Oftalmologia, é o método de aferição mais utilizado no mundo para medida da pressão intraocular (PIO), que se baseia no princípio de Imbert-Fick. Com isso, sofre influência de características da córnea, destacando-se a espessura ${ }^{(1,3)}$. De modo geral, assume-se que quanto mais fina seja a córnea, menor será sua resistência, causando uma menor medida da pressão, ou seja, valor hipoestimado. Por outro lado, quanto mais grossa a córnea, maior seria sua resistência e maior a pressão medida, valor hiperestimado por meio da TAG. Além da espessura, outras características como a curvatura da córnea, também são reconhecidamente importantes, de modo que córneas mais planas tendem a apresentar maior resistência para a aplanação e gerar valores mais elevados ${ }^{(4,5)}$. Entretanto, a medida da pressão intraocular sofre influência da córnea de forma mais complexa. Em um modelo matemático, Liu e Roberts ${ }^{(5)}$ observaram uma maior influência de variações relacionadas com a tensão da superfície pela resistência da córnea (módulo de Young), potencialmente determinando maior "erro" na TAG, comparando-se com os efeitos relacionados com a espessura central e com a ceratometria.

O estudo das propriedades biomecânicas da córnea, tema de crescente interesse e objetivo de importantes estudos relacionados com córnea, glaucoma e cirurgia refrativa, era limitado a modelos matemáticos e estudos experimentais até a introdução do Ocular Response Analyzer (ORA Reichert), em 2005 por Luce, $\mathrm{PhD}^{(6)}$.O ORA monitora dinamicamente a resposta de aplanamento da córnea pelo jato de ar, de modo a determinar propriedades biomecânicas da córnea, juntamente com as medidas da pressão intraocular. Além da medida da PIO calibrada de acordo com o "padrão ouro", TAG (IOPg - gold standard, Goldmann correlated Intraocular pressure), existe um cálculo para determinar uma pressão compensada da córnea de acordo com as propriedades biomecânicas encontradas (IOPcc - corneal compensated Intraocular pressure). Além das medidas pressóricas, IOPg e IOPcc, o ORA determina a histerese corneana $(\mathrm{CH}$ - Corneal Hysteresis) que é um parâmetro relacionado com a viscoelasticidade da córnea e o fator de resistência corneana (CRF - Corneal Resistance Factor $)^{(6-9)}$.

\section{Métodos}

Foram avaliados 47 olhos de 36 pacientes com diagnóstico de catarata que foram submetidos à cirurgia de facoemulsificação com implante de lente intraocular.

O estudo foi desenvolvido respeitando as normas e orientações estabelecidas na Declaração de Helsinki e as orientações e normas do Comitê de Ética em Pesquisa da Faculdade de Medicina da Universidade Federal Fluminense o qual aprovou sua execução.

Foram critérios de exclusão do estudo a presença de quaisquer doenças do segmento anterior ou diagnóstico prévio de glaucoma, suspeita de glaucoma e hipertensos oculares.

Todas as cirurgias foram realizadas pelo mesmo cirurgião, utilizando a incisão córnea clara $(2.7 \mathrm{~mm})$, agente viscoelástico (metilcelulose a $2 \%$ e $4 \%$ ), técnica Stop \& Chop e implante de lente intraocular (LIO) dobrável.

Após o exame oftalmológico de rotina, todos os pacientes inclusos no estudo foram avaliados pelo ORA (Ocular Response Analyzer, Reichert Ophthalmic Instruments, Buffalo, NY, USA) no pré-operatório, no primeiro e trigésimo dia de pós-operatório.

A figura 1 mostra o Sistema de não contato ORA (Ocular Response Analyzer).

O Teste de Kolmogorov-Smirnov foi utilizado para avaliar a distribuição normal. Diferenças obtidas entre as medidas obtidas através do Ocular Response Analyzer foram avaliados pelo T-test (Student's paired t-test). Foi considerado como estatisticamente significante $\mathrm{p}<0,05 \%$.

\section{$\underline{\text { Resultados }}$}

A média da IOPcc foi de $15.6( \pm 3.06)$ no préoperatório, de 15.5 (3.14) no primeiro dia após cirurgia e de $15.71( \pm 4.34)$ no trigésimo dia após facoemulsificação. A média de IOPg no pré-operatório foi de $14.7( \pm 3.0)$, no primeiro dia após cirurgia $12.9( \pm$ $3.1)$ e no trigésimo dia $13.4( \pm 3.7)$.

Tabela 1

Evolução da médias de IOPg e IOPcc

PRÉ $\quad$ D1 $\quad$ D30

\begin{tabular}{lrrr}
\hline IOPg & $14.7( \pm 3.0)$ & $12.9( \pm 3.1)$ & $13.4( \pm 3.7)$ \\
IOPcc & $15.6( \pm 3.06)$ & $15.5(3.14)$ & $15.7( \pm 4.34)$ \\
\hline
\end{tabular}




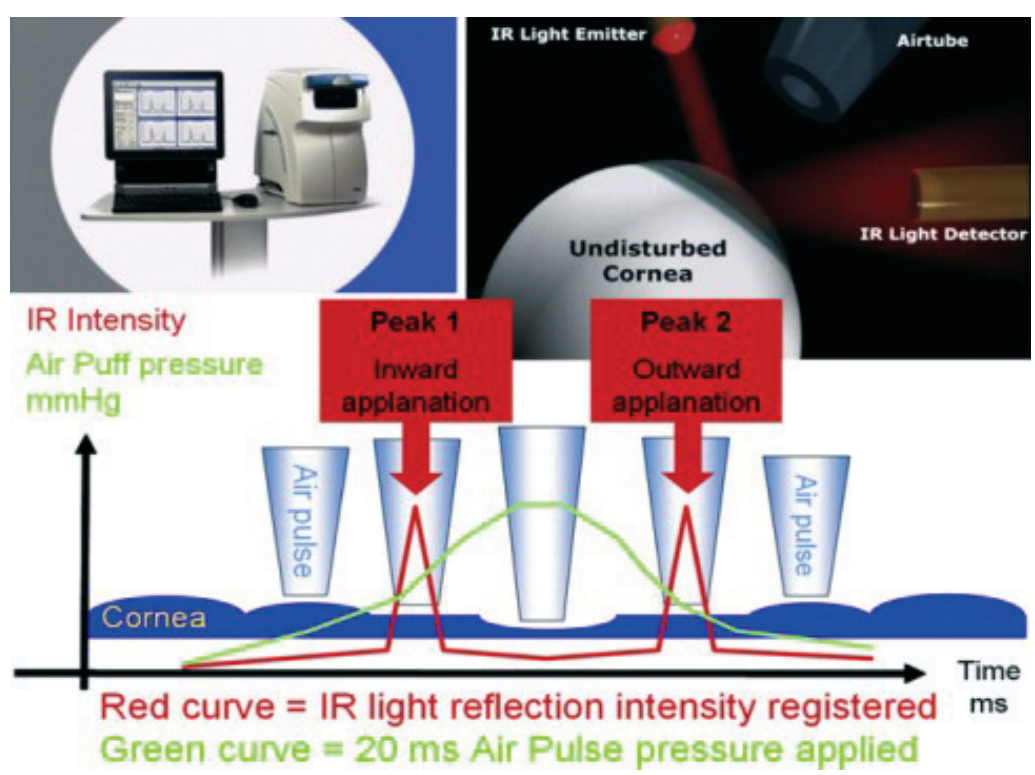

Figura 1: Sistema de Sopro de Ar de Não Contato - ORA (Ocular Response Analyzer)

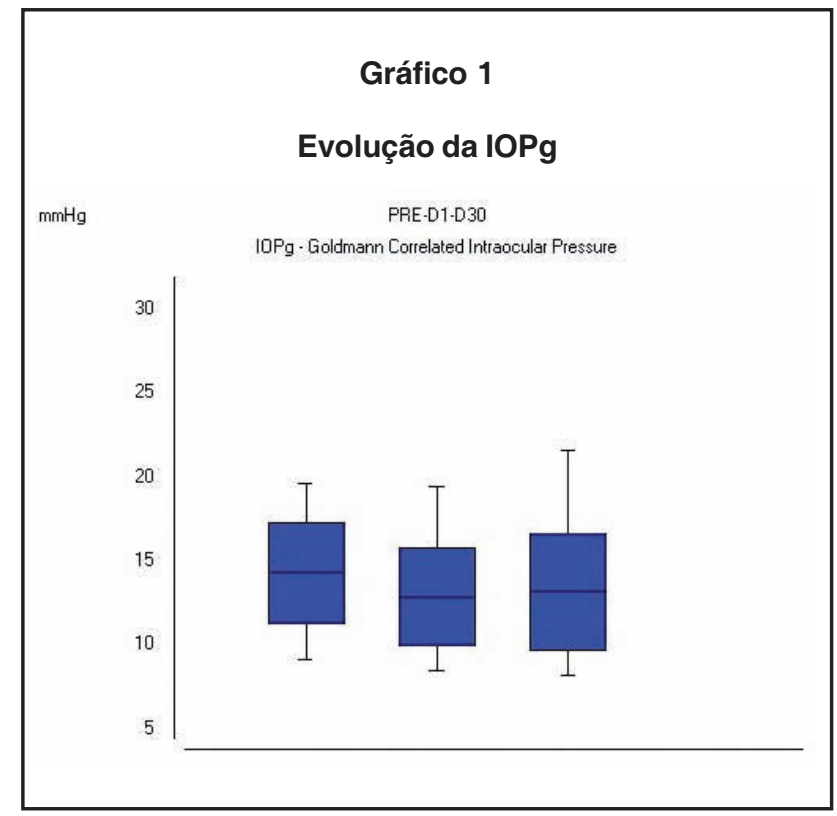

Podemos observar na tabela 1 a evolução das médias de IOPg e IOPcc e nos gráficos 1 e 2, demonstramos a evolução destas variáveis durante o tempo, respectivamente.

Houve diferença estatisticamente significativa entre IOPg (PRE X D1) com $p<0,01$. Supomos que o aumento da espessura corneana provocado pelo edema no primeiro dia pós-operatório influencia a IOPg, subestimando assim a medida da PIO nestes casos.

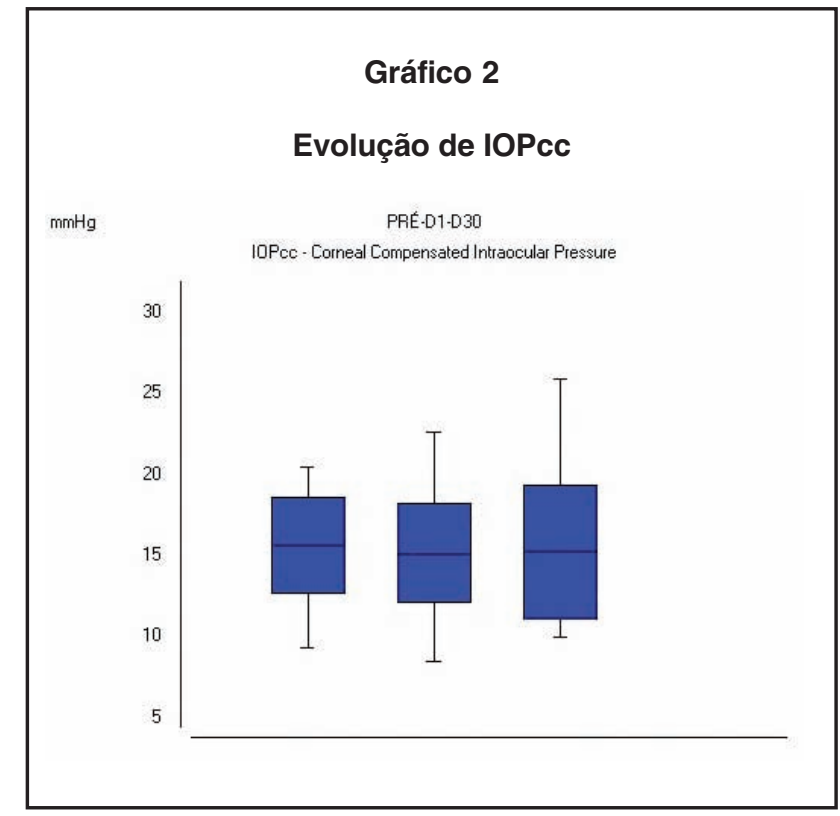

\section{Dıscussão}

Hipertensão ocular é uma complicação reconhecida após cirurgia de catarata, mesmo quando realizada sem complicações ou intercorrências per-operatórias ${ }^{(10,11)}$. A diminuição das propriedades biomecânicas da córnea está relacionada a dificuldade na capacidade de detectar o aumento da $\mathrm{PIO}^{(12,14)}$. Uma redução da $\mathrm{CH}$ e CRF foi encontrada após cirurgia da catarata, devido ao edema pós-operatório ${ }^{(12-14)}$. Em publicação recente, demonstra- 
mos que podemos detectar com o ORA, pressões intraoculares subestimadas pela Tonometria de Aplanação de Goldmann (padrão-ouro como método de aferição) ${ }^{(15)}$.Uma forma alternativa de se detectar tal aumento seria a tonometria de contorno dinâmico (PASCAL), que sofre menor influência das características da córnea por não ser necessário aplanação, mas apenas contato do sensor pressórico com a superfície $e^{(9,16-17)}$.

Com a introdução do ORA, iniciamos uma nova fase no entendimento das propriedades biomecânicas da córnea $^{(6)}$. Até então, o conhecimento nesta área era limitado a estudos experimentais e modelos matemáticos ${ }^{(8)}$.

Em um modelo matemático, Liu e Roberts ${ }^{(5)}$, observaram uma maior influência de variações relacionadas com a tensão da superfície relacionadas pela resistência da córnea (módulo de Young), potencialmente determinando maior "erro" naTAG, comparando-se com os efeitos relacionados com a espessura central e com a ceratometria.

Além da medida da PIO calibrada de acordo com o "padrão ouro",TAG (IOPg - gold standard, Goldmann correlated Intraocular pressure), existe um cálculo para determinar uma pressão compensada da córnea de acordo com as propriedades biomecânicas encontradas (IOPcc - corneal compensated Intraocular pressure). Além das medidas pressóricas, IOPg e IOPcc, o ORA determina a histerese corneana $(\mathrm{CH}$ - Corneal Hysteresis) que é um parâmetro relacionado com a visco-elasticidade da córnea e o fator de resistência corneana (CRF - Corneal Resistance Factor) ${ }^{(6-9)}$.

Podemos observar, neste artigo uma diminuição da IOPg (Goldmann correlated Intraocular pressure) no pósoperatório imediato pela medida do Ocular Response Analyzer, em córneas com aumento de espessura, devido a edema pós-facoemulsificação, isto ocorre possivelmente pela diminuição das propriedades biomecânicas $(\mathrm{CH}$ e CRF) no pós-operatório imediato ${ }^{(14)}$, podendo assim nos levar a equivocadas interpretações da real pressão intraocular nestes pacientes submetidos à facoemulsificação. Logo, devemos ressaltar a importância da Biomecânica da córnea na medida da pressão intraocular.

\section{Abstract}

Purpose: To evaluate the IOPg (gold standard, Goldmann correlated Intraocular pressure) and IOPcc (corneal compensated Intraocular pressure) in patients prior to and following phacoemulsification with intraocular lens implantation Methods: This is a prospective study. Thirty six consecutive patients (47 eyes) that underwent phacoemulsification surgery and intraocular lens implantation were enrolled. Full ophthalmologic clinical assessment including Ocular Response Analyzer (ORA, Reichert Ophthalmic Instruments, Buffalo, NY, USA) measurements were performed in all cases prior to surgery (Pre) and at follow-up time points: day 1 and day 30. Exclusion criteria were corneal disease or scars, previous ocular surgery and patients with glaucoma or glaucoma suspect or ocular hypertension. Student's paired t-test was utilized. Results: Mean and standard-deviation of ORA parameters were: IOPcc Pre was $15.6( \pm 3.06)$, day 1 (D1) $15.5( \pm 3.14)$ and day 30 (D30) $15.71( \pm 4.34)$. The mean of IOPg was Pre 14.7 ( \pm 3.0), day 1 (D1) 12.9 ( \pm 3.1), and day 30 (D30) was 13.4 ( \pm 3.7$)$. Conclusion: The reduction in the IOPg was observed in the immediate postoperative underestimating the real intraocular pressure ocular. We agree that biomechanical properties may have greater impact on IOP measurements error than corneal tchikness or curvature.

Keywords: Biomechanics; Intraocular pressure/ physiology; Cornea/physiology; Phacoemulsification; Diagnostic techniques, ophthalmological

\section{ReFERÊNCIAS}

1. Goldmann H, Schmidt T. [Applanation tonometry] Ophthalmologica. 1957;134(4):221-42. German.

2. Doughty MJ, Zaman ML. Human corneal thickness and its impact on intraocular pressure measures: a review and metaanalysis approach. Surv Ophthalmol. 2000;44(5):367-408.

3. Dueker DK, Singh K, Lin SC, Fechtner RD, Minckler DS, Samples JR, Schuman JS. Corneal thickness measurement in the management of primary open-angle glaucoma: a report by the American Academy of Ophthalmology. Ophthalmology. 2007;114(9):1779-87.

4. Sánchez-Tocino H, Bringas-Calvo R, Iglesias-Cortiñas D. [Correlation between intraocular pressure, paquimetry and keratometry in a normal population]. Arch Soc Esp Oftalmol. 2007;82(5):267-72. Spanish.

5. Liu J, Roberts CJ. Influence of corneal biomechanical properties on intraocular pressure measurement: quantitative analysis. J Cataract Refract Surg. 2005;31(1):146-55. Comment in: J Cataract Refract Surg. 2006;32(7):1073-4; author reply 1074 .

6. Luce DA. Determining in vivo biomechanical properties of the cornea with an ocular response analyzer. J Cataract $\mathrm{Re}$ fract Surg. 2005;31(1):156-62.

7. Kotecha A, Elsheikh A, Roberts CR, Zhu H, Garway-Heath DF. Corneal thickness- and age-related biomechanical properties of the cornea measured with the ocular response analyzer. Invest Ophthalmol Vis Sci. 2006;47(12):5337-47.

8. Alonso RS, Ventura MP, Ambrósio Jr R. Aspectos práticos da avaliação biomecânica da córnea. In: Vieira Netto M, Ambrósio Júnior R, Schor P, Chalita MR, Chamon W, editores. Wavefront, topografia e tomografia da córnea e segmento anterior: atualização propedêutica em cirurgia refrativa. Rio de Janeiro: Cultura Médica; 2006. 
9. Pepose JS, Feigenbaum SK, Qazi MA, Sanderson JP, Roberts CJ. Changes in corneal biomechanics and intraocular pressure following LASIK using static, dynamic, and noncontact tonometry. Am J Ophthalmol. 2007;143(1):39-47.

10. Alves MC, Faria RM, Raskin E, Raskin DG, Eredia GC, Andrade BBA. Hipertensão intraocular após facoemulsificação e implante de lente intraocular. Rev Bras Oftalmol. 2001;60(6):432-6.

11. Dayanir V, Ozcura F, Kir E, Topaloglu A, Ozkan SB, Aktunç T. Medical control of intraocular pressure after phacoemulsification. J Cataract Refract Surg. 2005;31(3):484-8.

12. Hager A, Loge K, Füllhas MO, Schroeder B, Grossherr M, Wiegand W. Changes in corneal hysteresis after clear corneal cataract surgery. Am J Ophthalmol. 2007;144(3):341-6.

13. Kucumen RB, Yenerel NM, Gorgun E, Kulacoglu DN, Oncel B, Kohen MC, Alimgil ML. Corneal biomechanical properties and intraocular pressure changes after phacoemulsification and intraocular lens implantation. J Cataract Refract Surg. 2008;34(12):2096-8. Comment in: J Cataract Refract Surg. 2009;35(11):2033-4; author reply 2034-5.

14. Valbon B, Ventura MP, Salomão M, Ambrósio R Jr. Correlation between central corneal thickness and biomechanical properties after phacoemulsification. Invest Ophthalmol Vis Sci. 2008;49:E-Abstract 656.
15. Valbon BF, Guerra F, Silva RS, Canedo AL, Ambrosio Júnior R. Hipertensão ocular "mascarada" por edema de córnea após cirurgia de catarata. Rev Bras Oftalmol. 2009;68(6):348-54.

16. Hager A, Loge K, Schroeder B, Füllhas MO, Wiegand W. Effect of central corneal thickness and corneal hysteresis on tonometry as measured by dynamic contour tonometry, ocular response analyzer, and Goldmann tonometry in glaucomatous eyes. J Glaucoma. 2008;17(5):361-5. Erratum in: J Glaucoma. 2008;17(7):604. Annette, Hager [corrected to Hager, Annette]; Kristina, Loge [corrected to Loge, Kristina]; Bernd, Schroeder [corrected to Schroeder, Bernd]; MarkOliver, Füllhas [corrected to Füllhas, Mark-Oliver]; Wolfgang, Wiegand [corrected to Wiegand, Wolfgang].

17. Boehm AG, Weber A, Pillunat LE, Koch R, Spoerl E. Dynamic contour tonometry in comparison to intracameral IOP measurements. Invest Ophthalmol Vis Sci. 2008;49(6):2472-7.
Endereço para correspondência
Bruno de Freitas Valbon
Rua Conde de Bonfim 211 apto. 712 - Tijuca
CEP 20.520-050 - Rio de Janeiro (RJ), Brasil
Tel: 55 (21) 8103-7117
E-mail: valbonbruno@ig.com.br 